\title{
AGM, Ranking Theory, and the Many Ways to Cope with Examples
}

\author{
Wolfgang Spohn
}

\begin{abstract}
The paper first explains how the ranking-theoretic belief change or conditionalization rules entail all of the standard AGM belief revision and contraction axioms. Those axioms have met a lot of objections and counter-examples, which thus extend to ranking theory as well. The paper argues for a paradigmatic set of cases that the counter-examples can be well accounted for with various pragmatic strategies while maintaining the axioms. So, one point of the paper is to save AGM belief revision theory as well as ranking theory. The other point, however, is to display how complex the pragmatic interaction of belief change and utterance meaning may be; it should be systematically and not only paradigmatically explored.
\end{abstract}

Keywords Ordinal conditional function - Ranking theory · AGM - Success postulate - Preservation postulate $\cdot$ Superexpansion postulate $\cdot$ Intersection postulate $\cdot$ Recovery postulate

\section{Introduction $^{1}$}

Expansions, revisions, and contractions are the three kinds of belief change intensely studied by AGM belief revision theory and famously characterized by the standard eight revision and eight contraction axioms. Even before their canonization in Alchourrón et al. (1985), ranking theory and its conditionalization rules for belief change (Spohn 1983, Sect. 5.3) generalized upon the AGM treatment. I always took the fact that these conditionalization rules entail the standard AGM axioms (as

\footnotetext{
1 I am grateful to Paul Arthur Pedersen for discussing his example in Sect. 6 with me, to David Makinson and Brian Leahy for various hints and corrections, and to two anonymous referees for further valuable remarks considerably improving the paper.
}

W. Spohn $(\varangle)$

Department of Philosophy, University of Konstanz, 78457 Konstanz, Germany

e-mail: wolfgang.spohn@uni-konstanz.de 
first observed in Spohn (1988), footnote 20, and in Gärdenfors (1988), Sect. 3.7) as reversely confirming ranking theory.

As is well known, however, a vigorous discussion has been going on in the last 20 years about the adequacy of those axioms, accumulating a large number of plausible counter-examples, which has cast a lot of doubt on the standard AGM theory and has resulted in a host of alternative axioms and theories. Via the entailment just mentioned these doubts extend to ranking theory; if those axioms fall, ranking theory falls, too. Following Christian Morgenstern's saying "weil nicht sein kann, was nicht sein darf", this paper attempts to dissolve those doubts by providing ranking-theoretic ways of dealing with those alleged counter-examples, which avoid giving up the standard AGM axioms. So, this defense of the standard AGM axioms is at the same time a self-defense of ranking theory.

This is the obvious goal of this paper. It is a quite restricted one, insofar as it exclusively focuses on those counter-examples. No further justification of AGM or ranking theory, no further comparative discussion with similar theories is intended; both are to be found extensively, if not exhaustively in the literature.

There is, however, also a mediate and no less important goal: namely to demonstrate the complexities of the pragmatic interaction between belief change and utterance meaning. I cannot offer any account of this interaction. Instead, the variety of pragmatic strategies I will be proposing in dealing with these examples should display the many aspects of that interaction that are hardly captured by any single account. So, one conclusion will be that this pragmatics, which has been little explored so far, should be systematically studied. And the other conclusion will be that because of those complexities any inference from such examples to the shape of the basic principles of belief change is premature and problematic. Those principles must be predominantly guided by theoretical considerations, as they are in both AGM and ranking theory in well-known ways.

The plan of the paper is this: I will recapitulate the basics of ranking theory in Sect. 2 and its relation to AGM belief revision theory in Sect. 3, as far as required for the subsequent discussion. There is no way of offering a complete treatment of the problematic examples having appeared in the literature. I have to focus on some paradigms, and I can only hope to have chosen the most important ones. I will first attend to revision axioms: Sect. 4 will deal with the objections against the Success Postulate, Sect. 5 with the Preservation Postulate, and Sect. 6 with the Superexpansion Postulate. Then I will turn to contraction axioms: Sect. 7 will be devoted to the Intersection Postulate, and Sect. 8 to the Recovery Postulate, perhaps the most contested one of all. Section 9 will conclude with a brief moral.

\section{Basics of Ranking Theory}

AGM belief revision theory is used to work with sentences of a given language $\mathbf{L}$ - just a propositional language; quantifiers and other linguistic complications are rarely considered. For the sake of simplicity let us even assume $\mathbf{L}$ to be finite, i.e., 
to have only finitely many atomic sentences. $\mathbf{L}$ is accompanied by some logic as specified in the consequence relation $\mathrm{Cn}$, which is usually taken to be the classical compact Tarskian entailment relation. I will assume it here as well (although there are variations we need not go into). A belief set is a deductively closed set of sentences of $\mathbf{L}$, usually a consistent one (since there is only one inconsistent belief set). Belief change then operates on belief sets. That is, expansion, revision, and contraction by $\varphi \in \mathbf{L}$ operate on belief sets; they carry a given belief set into a, respectively, expanded, revised, or contracted belief set.

By contrast, ranking theory is used to work with a Boolean algebra (or field of sets) $\mathscr{A}$ of propositions over a space $W$ of possibilities. Like probability measures, ranking functions are defined on such an algebra. Let us again assume the algebra $\mathscr{A}$ to be finite; the technical complications and variations arising with infinite algebras are not relevant for this paper (cf. Huber 2006; Spohn 2012, Chap. 5). Of course, the two frameworks are easily intertranslatable. Propositions simply are truth conditions of sentences, i.e., sets of valuations of $\mathbf{L}$ (where we may take those valuations as the possibilities in $W$ ). And if $T(\varphi)$ is the truth condition of $\varphi$, i.e., the set of valuations in which $\varphi$ is true, then $\{T(\varphi) \mid \varphi \in \mathbf{L}\}$ is an algebra-indeed a finite one, since we have assumed $\mathbf{L}$ to be finite.

I have always found it easier to work with propositions. For instance, logically equivalent sentences, which are not distinguished in belief revision theory, anyway (due to its extensionality axiom), reduce to identical propositions. And a belief set may be represented by a single proposition, namely as the intersection of all the propositions corresponding to the sentences in the belief set. The belief set is then recovered as the set of all sentences corresponding to supersets of that intersection in the algebra (since the classical logical consequence between sentences simply reduces to set inclusion between propositions).

Let me formally introduce the basic notions of ranking theory before explaining their standard interpretation:

Definition 1: $\kappa$ is a negative ranking function for $\mathscr{A}$ iff $\kappa$ is a function from $\mathscr{A}$ into $\mathbf{N}^{+}=\mathbf{N} \cup\{\infty\}$ such that for all $A, B \in \mathscr{A}$

(1) $\kappa(W)=0$,

(2) $\kappa(\varnothing)=\infty$, and

(3) $\kappa(A \cup B)=\min \{\kappa(A), \kappa(B)\}$.

Definition 2: $\beta$ is a positive ranking function for $\mathscr{A}$ iff $\beta$ is a function from $\mathscr{A}$ into $\mathbf{N}^{+}$such that for all $A, B \in \mathscr{A}$

(4) $\beta(\varnothing)=0$,

(5) $\beta(W)=\infty$, and

(6) $\beta(A \cap B)=\min \{\beta(A), \beta(B)\}$.

Negative and positive ranking functions are interdefinable via the equations:

(7) $\beta(A)=\kappa(\bar{A})$ and $\kappa(A)=\beta(\bar{A})$. A further notion that is often useful is this: 
Definition 3: $\tau$ is a two-sided ranking function for $\mathscr{A}$ (corresponding to $\kappa$ and/or $\beta$ ) iff

(8) $\tau(A)=\kappa(\bar{A})-\kappa(A)=\beta(A)-\kappa(A)$.

The axioms immediately entail the law of negation:

(9) either $\kappa(A)=0$ or $\kappa(\bar{A})=0$, or both (for negative ranks), and

(10) either $\beta(A)=0$ or $\beta(\bar{A})=0$, or both (for positive ranks), and

(11) $\tau(\bar{A})=-\tau(A)$ (for two-sided ranks).

Definition 4: Finally, the core of a negative ranking function $\kappa$ or a positive ranking function $\beta$ is the proposition

(12) $C=\bigcap\{A \mid \kappa(\bar{A})>0\}=\bigcap\{A \mid \beta(A)>0\}$.

Given the finiteness of $\mathscr{A}$ (or a strengthening of axioms (3) and (6) to infinite disjunctions or, respectively, conjunctions), we obviously have $\beta(C)>0$.

The standard interpretation of these notions is this: Negative ranks express degrees of disbelief. (Thus, despite being non-negative numbers, they express something negative and are therefore called negative ranks.) To be a bit more explicit, for $A \in \mathscr{A} \quad \kappa(A)=0$ says that $A$ is not disbelieved, and $\kappa(A)=n>0$ says that $A$ is disbelieved (to degree $n$ ). Disbelieving is taking to be false and believing is taking to be true. Hence, belief in $A$ is the same as disbelief in $\bar{A}$ and thus expressed by $\kappa(\bar{A})>0$. Note that we might have $\kappa(A)=\kappa(\bar{A})=0$, so that $A$ is neither believed nor disbelieved.

Positive ranks express degrees of belief directly. That is, $\beta(A)=0$ iff $A$ is not believed, and $\beta(A)=n>0$ iff $A$ is believed or taken to be true (to degree $n$ ). This interpretation of positive and negative ranks entails, of course, their interdefinability as displayed in (7).

Because of the axioms (1) and (4) beliefs are consistent; not everything is believed or disbelieved. Because of the axioms (3) and (6) beliefs are deductively closed. And the core of $\kappa$ or $\beta$ represents all those beliefs, by being their conjunction and entailing all of them and nothing else.

Finally, two-sided ranks are useful because they represent belief and disbelief in a single function. Clearly, we have $\tau(A)>0,<0$, or $=0$, iff, respectively, $A$ is believed, disbelieved, or neither. However, a direct axiomatization of two-sided ranks is clumsy; this is why I prefer to introduce them via Def. 3. Below I will freely change between negative, positive, and two-sided ranks.

As already indicated, ranks represent not only belief, but also degrees of belief; the $\operatorname{larger} \beta(A)$, the firmer your degree of belief in $A$. So, they offer an alternative model of such degrees. The standard model is probability theory, of course. However, it is very doubtful whether probabilities are able to represent beliefs, as the huge discussion triggered by the lottery paradox shows. (The lottery paradox precisely shows that axiom (c) of Def. 2 cannot be recovered in probabilistic terms.) So I consider it an advantage of ranking theory that it can represent both, beliefs and degrees of belief. (For all this see Spohn 2012, Chaps. 5 and 10.) 
Indeed, these degrees are cardinal, not ordinal (like Lewis' similarity spheres or AGM's entrenchment ordering), and they are accompanied by a measurement theory, which proves them to be measurable on a ratio scale (cf. Hild and Spohn 2008; Spohn 2012, Chap. 8). (Probabilities, by contrast, are usually measured on an absolute scale.)

I should perhaps mention that there are some formal variations concerning the range of ranking functions, which might consist of real or ordinal numbers instead of natural numbers; indeed, the measurement theory just mentioned works with real-valued ranking functions. In the infinite case, there is also some freedom in choosing the algebraic framework and in strengthening axioms (3) and (6). Here, we need not worry about such variations; it suffices to consider only the finite case and integer-valued ranking functions.

The numerical character of ranks is crucial for the next step of providing an adequate notion of conditional belief. This is generated by the notion of conditional ranks, which is more naturally defined in terms of negative ranking functions:

Definition 5: The negative conditional rank $\kappa(B \mid A)$ of $B \in \mathscr{A}$ given or conditional on $A \in \mathscr{A}$ (provided $\kappa(A)<\infty$ ) is defined by:

(13) $\kappa(B \mid A)=\kappa(A \cap B)-\kappa(A)$.

This is equivalent to the law of conjunction:

(14) $\kappa(A \cap B)=\kappa(A)+\kappa(B \mid A)$.

This law is intuitively most plausible: How strongly do you disbelieve $A \cap B$ ? Well, $A$ might be false; then $A \cap B$ is false as well; so take $\kappa(A)$, your degree of disbelief in $A$. But if $A$ should be true, $B$ must be false in order $A \cap B$ to be false. So add $\kappa(B \mid A)$, your degree of disbelief in $B$ given $A$.

The positive counterpart is the law of material implication:

(15) $\beta(A \rightarrow B)=\beta(B \mid A)+\beta(\bar{A})$-where $A \rightarrow B=\bar{A} \cup B$ is (set-theoretic) material implication and where the positive conditional rank $\beta(B \mid A)$ of $B$ given $A$ is defined in analogy to (7) by:

(16) $\beta(B \mid A)=\kappa(\bar{B} \mid A)$.

(15) is perhaps even more plausible: Your degree of belief in $A \rightarrow B$ is just your degree of belief in its vacuous truth, i.e., in $\bar{A}$, plus your conditional degree of belief in $B$ given $A$. This entails that your conditional rank and your positive rank of the material implication coincide if you don't take $A$ to be false, i.e., $\beta(\bar{A})=0$.

It should be obvious, though, that conditional ranks are much more tractable in negative than in positive terms. In particular, despite the interpretational differences there is a far-reaching formal analogy between ranks and probabilities. However, this analogy becomes intelligible only in terms of negative ranks and their axioms (1)-(3) and (13). This is why I have always preferred negative ranks to their positive counterparts.

Conditional ranks finally entail a notion of conditional belief:

(17) $B$ is conditionally believed given $A$ iff $\beta(B \mid A)=\kappa(\bar{B} \mid A)>0$. 
One further definition will be useful:

Definition 6: The negative ranking function $\kappa$ is regular iff for all $A \in \mathscr{A}$ with $A \neq \varnothing \kappa(A)<\infty$.

Hence, in a regular ranking function only the contradiction is maximally firmly disbelieved, and only the tautology is maximally firmly believed. And conditional ranks are universally defined except for the contradictory condition. This corresponds to the probabilistic notion of regularity.

There is no space for extensive comparative observations. Just a few remarks: Ranking functions have ample precedent in the literature, at least in Shackle's (1961) functions of potential surprise, Rescher's (1964) hypothetical reasoning, and Cohen's (1970) functions of inductive support. All these predecessors arrived at the Baconian structure of (1)-(3) or (4)-(6), as it is called by Cohen (1980). However, none of them has an adequate notion of conditional ranks as given by (13) or (16); this is the crucial advance of ranking theory (cf. Spohn 2012, Sect. 11.1).

AGM belief revision theory seems to adequately capture at least the notion of conditional belief. However, in my view it founders at the problem of iterated belief revision. The point is that conditional belief is there explained only via the ordinal notion of an entrenchment ordering, but within these ordinal confines no convincing account of iterated revision can be found. (Of course, the defense of this claim would take many pages.) The iteration requires the cardinal resources of ranking theory, in particular the cardinal notion of conditional ranks (cf. Spohn 2012, Chaps. 5 and 8).

Finally, ranking theory is essentially formally equivalent to possibility theory as suggested by Zadeh (1978), fully elaborated in Dubois and Prade (1988), and further expanded in many papers; the theories are related by an exponential (or logarithmic) scale transformation. However, while ranking theory was determinately intended to capture the notion of belief, possibility theory was and is less determinate in my view. This interpretational indecision led to difficulties in defining conditional degrees of possibility, which is not an intuitive notion, anyway, and therefore formally explicable in various ways, only one of which corresponds to (13) (cf. Spohn 2012, Sect. 11.8). AGM unambiguously talk about belief, and therefore I continue my discussion in terms of ranking theory, which does the same.

Above I introduced my standard interpretation of ranking theory, which I then extended to conditional belief. However, one should note that it is by no means mandatory. On this interpretation, there are many degrees of belief, many degrees of disbelief, but only one degree of unopinionatedness, namely the two-sided rank 0 . This looks dubious. However, we are not forced to this interpretation. We might as well take some threshold value $z>0$ and say that only $\beta(A)>z$ expresses belief. Or in terms of two-sided ranks: $\tau(A)>z$ is belief, $-z \leq \tau(A) \leq z$ is unopinionatedness, and $\tau(A)<-z$ is disbelief. Then, the basic laws of belief are still preserved, i.e., belief sets are always consistent and deductively closed. It's only that the higher the threshold $z$, the stricter the notion of belief. I take this to account for the familiar vagueness of the notion of belief; there is only a vague answer to the question: How firmly do you have to believe something in order to believe it? 
Still, the Lockean thesis ("belief is sufficient degree of belief") can be preserved in this way, while it must be rejected if degrees of belief are probabilities. Of course, the vagueness also extends to conditional belief. However, the ranking-theoretic apparatus underneath is entirely unaffected by that reinterpretation.

Let us call this the variable interpretation of ranking theory. Below, the standard interpretation will be the default. But at a few places, which will be made explicit, the variable interpretation will turn out to be useful.

\section{AGM Expansion, Revision, and Contraction as Special Cases of Ranking-Theoretic Conditionalization}

The notion of conditional belief is crucial for the next point. How do we change belief states as represented by ranking functions? One idea might be that upon experiencing $A$ we just move to the ranks conditional on $A$. However, this means treating experience as absolutely certain ( since $\beta(A \mid A)=\infty$ ); nothing then could cast any doubt on that experience. This is rarely or never the case; simple probabilistic conditionalization suffers from the same defect. This is why Jeffrey (1965/1983, Chap. 11) proposed a more general version of conditionalization, and in Spohn (1983, Sect. 5.3, 1988, Sect. 5) I proposed to transfer this idea to ranking theory:

Definition 7: Let $\kappa$ be a negative ranking function for $\mathscr{A}$ and $A \in \mathscr{A}$ such that $\kappa(A)$, $\kappa(\bar{A})<\infty$, and $n \in \mathbf{N}^{+}$. Then the $A \rightarrow n$-conditionalization $\kappa_{A \rightarrow n}$ of $\kappa$ is defined by

(18) $\kappa_{A \rightarrow n}(B)=\min \{\kappa(B \mid A), \kappa(B \mid \bar{A})+n\}$.

The $A \rightarrow n$-conditionalization will be called result-oriented.

It is easily checked that

(19) $\kappa_{A \rightarrow n}(A)=0$ and $\kappa_{A \rightarrow n}(\bar{A})=n$.

Thus, the parameter $n$ specifies the posterior degree of belief in $A$ and hence the result of the belief change; this is why I call it result-oriented. It seems obvious to me that learning must be characterized by such a parameter; the learned can be learned with more or less certainty. Moreover, for any $B$ we have $\kappa_{A \rightarrow n}(B \mid A)=\kappa(B \mid A)$ and $\kappa_{A \rightarrow n}(B \mid \bar{A})=\kappa(B \mid \bar{A})$. In sum, we might describe $A \rightarrow n$-conditionalization as shifting the $A$-part and the $\bar{A}$-part of $\kappa$ in such a way that $A$ and $\bar{A}$ get their intended ranks and as leaving the ranks conditional on $A$ and on $\bar{A}$ unchanged. This was also the crucial rationale behind Jeffrey's generalized conditionalization (cf. also Teller 1976).

However, as just noticed, the parameter $n$ specifies the effect of experience, but does not characterize experience by itself. This objection was also raised against Jeffrey_by Field (1978), who proposed quite an intricate way to meet it. In ranking terms the remedy is much simpler: 
Definition 8: As before, let $\kappa$ be a negative ranking function for $\mathscr{A}, A \in \mathscr{A}$ such that $\kappa(A), \kappa(\bar{A})<\infty$, and $n \in \mathbf{N}^{+}$. Then the $A \uparrow n$-conditionalization $\kappa_{A \uparrow n}$ of $\kappa$ is defined by

(20) $\kappa_{A \uparrow n}(B)=\min \{\kappa(A \cap B)-m, \kappa(\bar{A} \cap B)+n-m\}$, where $m=\min \{\kappa(A), n\}$.

The $A \uparrow n$-conditionalization will be called evidence-oriented.

The effect of this conditionalization is that, whatever the prior ranks of $A$ and $\bar{A}$, the posterior rank of $A$ improves by exactly $n$ ranks in comparison to the prior rank of $A$. This is most perspicuous in the easily provable equation

(21) $\tau_{A \uparrow n}(A)-\tau(A)=n$

for the corresponding two-sided ranking function. So, now the parameter $n$ indeed characterizes the nature and the strength of the evidence by itself-whence the name.

Of course, the two kinds of conditionalization are interdefinable; we have:

(22) $\kappa_{A \rightarrow n}=\kappa_{A \uparrow m}$, where $m=\tau(\bar{A})+n$.

Result-oriented conditionalization is also called Spohn conditionalization, since it was the version I proposed, whereas evidence-oriented conditionalization is also called Shenoy conditionalization, since it originates from Shenoy (1991). There are, moreover, generalized versions of each, where either the direct effect of learning or the experience itself is characterized by some ranking function on some partition of the given possibility space (not necessarily a binary partition $\{A, \bar{A}\}$ ), as already proposed by Jeffrey (1965/1983, Chap. 11) for probabilistic learning. This generalized conditionalization certainly provides the most general and flexible learning rule in ranking terms. However, there is no need to formally introduce it; below I will refer only to the simpler rules already stated. (For more careful explanations of this material see Spohn 2012, Chap. 5.)

All of this is directly related to AGM belief revision theory. First, these rules of conditionalization map a ranking function into a ranking function. Then, however, they also map the associated belief sets (= set of all propositions entailed by the relevant core). Thus, they do what AGM expansions, revisions, and contractions do. The latter may now easily be seen to be special cases of result-oriented conditionalization. At least, the following explications seem to fully capture the intentions of these three basic AGM movements:

Definition 9: Expansion by $A$ simply is $A \rightarrow n$-conditionalization for some $n>0$, provided that $\tau(A) \geq 0$; that is, the prior state is does not take $A$ to be false, and the posterior state believes or accepts $A$ with some firmness $n$.

Definition 10: Revision by $A$ is $A \rightarrow n$-conditionalization for some $n>0$, provided that $-\infty<\tau(A)<0$; that is, the prior state disbelieves $A$ and the posterior state is forced to accept $A$ with some firmness $n$. In the exceptional case where $\tau(A)=-\infty$ no $A \rightarrow n$-conditionalization and hence no revised ranking function is defined. In this case we stipulate that the associated belief set is the inconsistent one. With this stipulation, ranking-theoretic revision is as generally defined as AGM revision. 
For both, expansion and revision by $A$, it does not matter how large the parameter $n$ is, as long as it is positive. Although the posterior ranking function varies with different $n$, the posterior belief set is always the same; a difference in belief sets could only show up after iterated revisions.

As to contraction by $A: A \rightarrow 0$-conditionalization amounts to a two-sided contraction either by $A$ or by $\bar{A}$ (if one of these contractions is substantial, the other one must be vacuous); whatever the prior opinion about $A$, the posterior state then is unopinionated about $A$. Hence, we reproduce AGM contraction in the following way:

Definition 11: Contraction by $A$ is $A \rightarrow 0$-conditionalization in case $A$ is believed, but not maximally, i.e., $\infty>\tau(A)>0$, and as no change at all in case $A$ is not believed, i.e., $\tau(A) \leq 0$. In the exceptional case where $\tau(A)=\infty$, no $A \rightarrow 0$ conditionalization and hence no contracted ranking function is defined. In this case we stipulate that the contraction is vacuous and does not change the belief set. Thereby ranking-theoretic contraction is also as generally defined as AGM contraction.

It should be clear that these three special cases do not exhaust conditionalization. For instance, there is also the case where evidence directly weakens, though does not eliminate the disbelief in the initially disbelieved $A$. Moreover, evidence might also speak against $A$; but this is the same as evidence in favor of $\bar{A}$.

The crucial observation for the rest of the paper now is that revision and contraction thus ranking-theoretically defined entail all eight AGM revision and all eight AGM contractions axioms, $(\mathrm{K} * 1)-(\mathrm{K} * 8)$ and $(\mathrm{K} \div 1)-(\mathrm{K} \div 8)$-provided we restrict the ranking-theoretic operations to regular ranking functions. The effect of this assumption is that $\varnothing$ is the only exceptional case for revision and $W$ the only exceptional case for contraction.

For most of the axioms this entailment is quite obvious (for full details see Spohn 2012, Sect.5.5). In the sequel, I move to and fro between the sentential framework (using greek letters and propositional logic) and the propositional framework (using italics and set algebra). This should not lead to any misunderstanding. $K$ is a variable for belief sets, $K * \varphi$ denotes the revision of $K$ by $\varphi \in \mathbf{L}$ and $K \div \varphi$ the contraction of $K$ by $\varphi$. Finally $A=T(\varphi)$ and $B=T(\psi)$.

$(\mathrm{K} * 1)$, Closure, says: $K * \varphi=C n(K * \varphi)$. It is satisfied by Definiton 10, because, according to each ranking function, the set of beliefs is deductively closed.

$(\mathrm{K} * 2)$, Success, says in AGM terms: $\varphi \in K * \varphi$. With Def. 10 this translates into: $\kappa_{A \rightarrow n}(\bar{A})>0 \quad(n>0)$. This is true by definition (where we require regularity entailing that $\kappa_{A \rightarrow n}$ is defined for all $A \neq \emptyset$ ).

$(\mathrm{K} * 3)$, Expansion 1, says in AGM terms: $K * \varphi \subseteq C n(K \cup\{\varphi\})$.

$(\mathrm{K} * 4)$, Expansion 2, says: if $\neg \varphi \notin K$, then $C n(K \cup\{\varphi\}) \subseteq K * \varphi$. Together, $(K * 3)$ and $(K * 4)$ are equivalent to $K * \varphi=C n(K \cup\{\varphi\})$, provided that $\neg \varphi \notin K$. With Def. 10 this translates into: if $\kappa(A)=0$ and if $C$ is the core of $\kappa$, then the core of $\kappa_{A \rightarrow n}(n>0)$ is $C \cap A$. This is obviously true. 
(K*5), Consistency Preservation, says in AGM terms: if $\perp \notin C n(K)$ and $\perp \notin$ $C n(\varphi)$, then $\perp \notin K * \varphi$ ( $\perp$ is some contradictory sentence). This holds because, if $\kappa$ is regular, $\kappa_{A \rightarrow n}(n>0)$ is regular, too, and both have consistent belief sets.

$(\mathrm{K} * 6)$, Extensionality, says in AGM terms: if $C n(\varphi)=C n(\psi)$, then $K * \varphi=$ $K * \psi$. And in ranking terms: $\kappa_{A \rightarrow n}=\kappa_{A \rightarrow n}$. It is built into the propositional framework.

$(\mathrm{K} * 7)$, Superexpansion, says in AGM terms: $K *(\varphi \wedge \psi) \subseteq C n((K * \varphi) \cup\{\psi\})$.

$(\mathrm{K} * 8)$, Subexpansion, finally says: if $\neg \psi \notin K * \varphi$, then $C n((K * \varphi) \cup\{\psi\}) \subseteq$ $K *(\varphi \wedge \psi)$. In analogy to $(K * 3)$ and $(K * 4)$, the conjunction of $(K * 7)$ and $(K * 8)$ translates via Def. 10 into: if $\kappa(B \mid A)=0$ and if $C$ is the core of $\kappa_{A \rightarrow n}(n>0)$ then the core of $\kappa_{A \cap B \rightarrow n}$ is $C \cap B$. This is easily seen to be true. The point is this: Although Rott (1999) is right in emphasizing that $(\mathrm{K} * 7)$ and $(\mathrm{K} * 8)$ are not about iterated revision, within ranking theory they come to that, and they say then that $(\mathrm{K} * 3)$ and $(\mathrm{K} * 4)$ hold also after some previous revision; and, of course, $(\mathrm{K} * 3)$ and $(\mathrm{K} * 4)$ hold for any ranking function.

Similarly for the contraction axioms:

$(\mathrm{K} \div 1)$, Closure, says: $K \div \varphi=C n(K \div \varphi)$. It holds as trivially as $(\mathrm{K} * 1)$.

$(\mathrm{K} \div 2)$, Inclusion, says in AGM terms: $K \div \varphi \subseteq K$. And via Definition 11 in ranking terms: the core of $\kappa$ is a subset of the core of $\kappa_{A \rightarrow 0}$. This is indeed true by definition.

(K $\div 3$, Vacuity, says in AGM terms: if $\varphi \notin K$, then $K \div \varphi=K$. And in ranking terms: If $\kappa(\bar{A})=0$, then $\kappa_{A \rightarrow 0}=\kappa$. This is true by Definition 11 .

$(\mathrm{K} \div 4)$, Success, says in AGM terms: $\varphi \notin K \div \varphi$, unless $\varphi \in C n(\emptyset)$. And in ranking terms: if $A \neq W$, then $\kappa_{A \rightarrow 0}(A)=0$. Again this is true by Def. 11, also because $\kappa_{A \rightarrow 0}$ is defined for all $A \neq W$ due to the regularity of $\kappa$.

$(\mathrm{K} \div 5)$, Recovery, says in AGM terms: $K \subseteq C n((K \div \varphi) \cup\{\varphi\})$. With Def. 11 this translates into ranking terms: if $C$ is the core of $\kappa$ and $C^{\prime}$ the core of $\kappa_{A \rightarrow 0}$, then $C^{\prime} \cap A \subseteq C$. This holds because $C \subseteq C^{\prime}$ and $C^{\prime}-C \subseteq \bar{A}$.

$(\mathrm{K} \div 6)$, Extensionality, says: if $\operatorname{Cn}(\varphi)=C n(\psi)$, then $K \div \varphi=K \div \psi$. It is again guaranteed by our propositional framework.

$(\mathrm{K} \div 7)$, Intersection, says in AGM terms: $(K \div \varphi) \cap(K \div \psi) \subseteq K \div(\varphi \wedge \psi)$.

$(\mathrm{K} \div 8)$, Conjunction, finally says: if $\varphi \notin K \div(\varphi \wedge \psi)$, then $K \div(\varphi \wedge \psi) \subseteq K \div \varphi$. Both translate via Def. 11 into the corresponding assertions about the cores of the ranking functions involved. I spare myself showing their ranking-theoretic validity, also because of the next observation. (But see Spohn 2012, p. 90.)

As to the relation between AGM revision and contraction, I should add that the Levi Identity and the Harper Identity also hold according to the ranking-theoretic account of those operations:

The Levi Identity says in AGM terms: $K * \varphi=C n((K \div \neg \varphi) \cup\{\varphi\})$. And in ranking terms: if $C^{\prime}$ is the core of $\kappa_{A \rightarrow n}(n>0)$ and $C^{\prime \prime}$ the core of $\kappa_{\bar{A} \rightarrow 0}$, then $C^{\prime}=C^{\prime \prime} \cap A$. It thus reduces revision to contraction (and expansion) and is immediately entailed by Defs. 10-11.

The Harper Identity says in AGM terms: $K \div \varphi=K \cap(K * \neg \varphi)$. And in ranking terms: if $C$ is the core of $\kappa, C^{\prime}$ is the core of $\kappa_{\bar{A} \rightarrow n}(n>0)$, and $C^{\prime \prime}$ the core of $\kappa_{A \rightarrow 0}$, then $C^{\prime \prime}=C \cup C^{\prime}$. It thus reduces contraction to revision and holds again because of 
Def. 10-11. Moreover, since the Harper Identity translates the eight revision axioms $(\mathrm{K} * 1)-(\mathrm{K} * 8)$ into the eight contraction axioms $(\mathrm{K} \div 1)-(\mathrm{K} \div 8)$ and since ranking-theoretic revision satisfies $(\mathrm{K} * 1)-(\mathrm{K} * 8)$, as shown, ranking-theoretic contraction must satisfy $(\mathrm{K} \div 1)-(\mathrm{K} \div 8)$; so, this proves $(\mathrm{K} \div 7)-(\mathrm{K} \div 8)$.

I should finally add that the picture does not really change under the variable interpretation introduced at the end of the previous section. Only the variants of conditionalization increase thereby. I have already noted that expansion and revision are unique only at the level of belief sets, but not at the ranking-theoretic level. Under the variable interpretation, contraction looses its uniqueness as well, because under this interpretation there are also many degrees of unopinionatedness. However, rank 0 preserves its special status, since it is the only rank $n$ for which possibly $\tau(A)=$ $\tau(\bar{A})=n$. Hence, the unique contraction within the standard interpretation may now be called central contraction, which is still special.

The problem I want to address in this paper is now obvious. If many of the AGM revision and contraction postulates seem objectionable or lead to unintuitive results, then the above ranking-theoretic explications of AGM revision and contraction, which entail those postulates, must be equally objectionable. Hence, if I want to maintain ranking theory, I must defend AGM belief revision theory against these objections. This is what I shall do in the rest of this paper closely following Spohn (2012, Sect.11.3), and we will see that ranking theory helps enormously with this defense. I cannot cover the grounds completely. However, if my strategy works with the central objections to be chosen, it is likely to succeed generally.

\section{The Success Postulate for AGM-Revision}

Let me start with three of the AGM postulates for revision. A larger discussion originated from the apparently undue rigidity of the Success postulate $(K * 4)$ requiring that

(23) $\varphi \in K * \varphi$,

i.e., that the new evidence must be accepted. Many thought that "new information is often rejected if it contradicts more entrenched previous beliefs" (Hansson 1997, p. 2) or that if new information "conflicts with the old information in $K$, we may wish to weigh it against the old material, and if it is ... incredible, we may not wish to accept it" (Makinson 1997, p. 14). Thus, belief revision theorists tried to find accounts for what they called non-prioritized belief revision. Hansson (1997) is a whole journal issue devoted to this problem.

The idea is plausible, no doubt. However, the talk of weighing notoriously remains an unexplained metaphor in belief revision theory; and the proposals are too ramified to be discussed here. Is ranking theory able to deal with non-prioritized belief revision?

Yes. After all, ranking theory is made for the metaphor of weighing (cf. Spohn 2012, Sect. 6.3). So, how do we weigh new evidence against old beliefs? Above I 
explained revision by $A$ as result-oriented $A \rightarrow n$-conditionalization for some $n>0$ (as far as belief sets were concerned, the result was the same for all $n>0$ ). And thus Success was automatically satisfied. However, I also noticed that evidenceoriented $A \uparrow n$-conditionalization may be a more adequate characterization of belief dynamics insofar as its parameter $n$ pertains only to the evidence. Now we can see that this variant conditionalization is exactly suited for describing non-prioritized belief revision.

If we assume that evidence always comes with the same firmness $n>0$, then $A \uparrow n$-conditionalization of a ranking function $\kappa$ is sufficient for accepting $A$ if $\kappa$ $(A)<n$ and is not sufficient for accepting $A$ otherwise. One might object that the evidence $A$ is here only weighed against the prior disbelief in $A$. But insofar as the prior disbelief in $A$ is already a product of a weighing of reasons (as described in Spohn 2012, Sect.6.3), the evidence $A$ is also weighed against these old reasons. It is not difficult to show that $A \uparrow n$-conditionalization with a fixed $n$ is a model of screened revision as defined by Makinson (1997, p. 16). And if we let the parameter $n$ sufficient for accepting the evidence vary with the evidence $A$, we should also be able to model relationally screened revision (Makinson 1997, p. 19).

Was this a defense of Success and thus of AGM belief revision? Yes and no. The observation teaches the generality and flexibility of ranking-theoretic conditionalization. We may define belief revision within ranking theory in such a way as to satisfy Success without loss. But we also see that ranking theory provides other kinds of belief change which comply with other intuitive desiderata and which we may, or may not, call belief revision. In any case, ranking-theoretic conditionalization is broad enough to cover what has been called non-prioritized belief revision.

\section{The Preservation Postulate}

Another interesting example starts from the observation that $(\mathrm{K} * 4)$, Expansion 2, is equivalent to the Preservation postulate, given $(\mathrm{K} * 2)$, Success:

(24) if $\neg \varphi \notin K$, then $K \subseteq K * \varphi$

Preservation played an important role in the rejection of the unrestricted Ramsey test in Gärdenfors (1988, Sect.7.4). Later on it became clear that Preservation is wrong if applied to conditional sentences $\varphi$ (cf. Rott 1989) or, indeed, to any kind of auto-epistemic or reflective statements. Still, for sentences $\varphi$ in our basic language L, Preservation appeared unassailable.

Be this as it may, even Preservation has met intuitive doubts. Rabinowicz (1996) discusses the following simple story: Suppose that given all my evidence I believe that Paul committed a certain crime $(=\psi)$; so $\psi \in K$. Now a new witness turns up producing an alibi for Paul $(=\varphi)$. Rabinowicz assumes that $\varphi$, though surprising, might well be logically compatible with $K$; so $\neg \varphi \notin K$. However, after the testimony I no longer believe in Paul's guilt, so $\psi \notin K * \varphi$, in contradiction to Preservation. 


\begin{tabular}{c|c|c}
$\kappa$ & $\psi$ & $\neg \psi$ \\
\hline$\varphi$ & 3 & 6 \\
\hline$\neg \varphi$ & 0 & 9 \\
\hline
\end{tabular}

\begin{tabular}{c|c|c|}
$\kappa^{\prime}$ & $\psi$ & $\neg \psi$ \\
\hline$\varphi$ & 0 & 3 \\
\hline$\neg \varphi$ & 6 & 15 \\
\hline
\end{tabular}

Fig. 1 A Counter-example to Preservation?

Prima facie, Rabinowicz' assumptions seem incoherent. If I believe Paul to be guilty, I thereby exclude the proposition that any such witness will turn up; the appearance of the witness is a surprise initially disbelieved. So, we have $\neg \varphi \in K$ after all, and Preservation does not apply and holds vacuously.

Look, however, at the following negative ranking function $\kappa$ and its $\varphi \rightarrow 6$ - or $\varphi \uparrow 9$-conditionalization $\kappa^{\prime}$ (again, forgive me for mixing the sentential and the propositional framework) (Fig. 1).

As it should be, the witness is negatively relevant to Paul's guilt according to $\kappa$ (and vice versa); indeed, Paul's being guilty $(\psi)$ is a necessary and sufficient reason for assuming that there is no alibi $(\neg \varphi)$-in the sense that $\neg \varphi$ is believed given $\psi$ and $\varphi$ is believed given $\neg \psi$. Hence, we have $\kappa(\neg \psi)=6$, i.e., I initially believe in Paul's guilt, and confirming our first impression, $\kappa(\varphi)=3$, i.e., I initially disbelieve in the alibi.

However, I have just tacitly assumed the standard interpretation in which negative rank $>0$ is the criterion of disbelief. We need not make this assumption. I emphasized at the end of Sect. 2 that we might conceive disbelief more strictly according to the variable interpretation, say, as negative rank $>5$. Now note what happens in our numerical example: Since $\kappa(\neg \psi)=6$ and $\kappa(\varphi)=3$, I do initially believe in Paul's guilt, but not in the absence of an alibi (though one might say that I have positive inclinations toward the latter). Paul's guilt is still positively relevant to the absence of the alibi, but neither necessary nor sufficient for believing the latter. After getting firmly informed about the witness, I change to $\kappa^{\prime}(\neg \varphi)=6$ and $\kappa(\psi)=3$; that is, I believe afterwards that Paul has an alibi (even according to our stricter criterion of belief) and do not believe that he has committed the crime (though I am still suspicious).

By thus exploiting the vagueness of the notion of belief, we have found a model that accounts for Rabinowicz' intuitions. Moreover, we have described an operation that may as well be called belief revision, even though it violates Preservation. Still, this is not a refutation of Preservation. If belief can be taken as more or less strict, belief revision might mean various things and might show varying behavior. And the example has in fact confirmed that, under our standard interpretation (with disbelief being rank $>0$ ), belief revision should conform to preservation.

This raises an interesting question: What is the logic of belief revision (and contraction) under the variable interpretation of belief within ranking theory just used? I don't know; I have not explored the issue. What is clear is only that the logic of central contraction (cf. the end of Sect. 3) is the same as the standard logic of contraction, because central contraction is contraction under the standard interpretation. 


\section{The Superexpansion Postulate}

As already noticed by Gärdenfors (1988, p. 57), (K * 7), Superexpansion, is equivalent to the following assertion, given $(\mathrm{K} * 1)-(\mathrm{K} * 6)$ :

(25) $K * \varphi \cap K * \psi \subseteq K *(\varphi \vee \psi)$.

Arthur Paul Pedersen has given the following very plausible example that is at least a challenge to that assertion (quote from personal communication):

Tom is president of country $X$. Among other things, Tom believes

$\neg \varphi$ : Country $A$ will not bomb country $X$.

$\neg \psi$ : Country $B$ will not bomb country $X$.

Tom is meeting with the chief intelligence officer of country $X$, who is competent, serious, and honest.

Scenario 1: The intelligence officer informs Tom that country $A$ will bomb country $X(\varphi)$. Tom accordingly believes that country $A$ will bomb country $X$, but he retains his belief that country $B$ will not bomb country $X(\neg \psi)$. Because Tom's beliefs are closed under logical consequence, Tom also believes that either country $A$ or country $B$ will not bomb country $X$ $(\neg \varphi \vee \neg \psi)$.

So $\varphi, \neg \psi, \neg \varphi \vee \neg \psi$ are in $K * \varphi$.

Scenario 2: The intelligence officer tells Tom that country $B$ will bomb country $X(\psi)$. Tom accordingly believes that country $B$ will bomb country $X$, but he retains his belief that country $A$ will not bomb country $X(\neg \varphi)$. Because Tom's beliefs are closed under logical consequence, Tom also believes that either country $A$ or country $B$ will not bomb country $X$ $(\neg \varphi \vee \neg \psi)$.

$$
\text { So } \psi, \neg \varphi, \neg \varphi \vee \neg \psi \text { are in } K * \psi \text {. }
$$

Scenario 3: The intelligence officer informs Tom that country $A$ or country $B$ or both will bomb country $X(\varphi \vee \psi)$. In this scenario, Tom does not retain his belief that country $A$ will not bomb country $X(\neg \varphi)$. Nor does Tom retain his belief that country $B$ will not bomb country $X(\neg \psi)$. Furthermore, Tom does not retain his belief that either country $A$ or country $B$ will not bomb country $X(\neg \varphi \vee \neg \psi)$ - that is to say, his belief that it is not the case that both country $A$ and country $B$ will bomb country $X$ - for he now considers it a serious possibility that both country $A$ and country $B$ will bomb country $X$. Accordingly, Tom accepts that country $A$ or country $B$ or both will bomb country $X(\varphi \vee \psi)$, but Tom retracts his belief that country $A$ will not bomb country $X(\neg \varphi)$, his belief that country $B$ will not bomb country $X(\neg \psi)$, and his belief that either country $A$ or country $B$ will not bomb country $X(\neg \varphi \vee \neg \psi)$.

So $\varphi \vee \psi$ is in $K *(\varphi \vee \psi)$.

Importantly, $\neg \varphi \vee \neg \psi$ is not in $K *(\varphi \vee \psi)$ !

One can understand the reason for the retraction of $\neg \varphi \vee \neg \psi$ in Scenario 3 as follows: If after having learned that either country $A$ or country $B$ will bomb country $X$ Tom learns that country $A$ will bomb country $X$, for him it is not settled whether country $B$ will bomb country $X$. Yet if Tom were to retain his belief that either country $A$ or country $B$ will not 


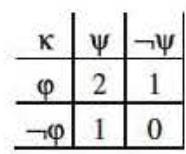

Initial

State
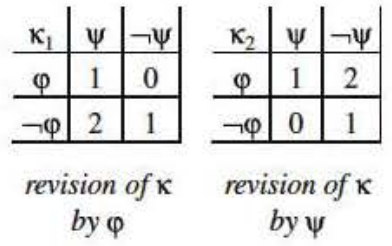

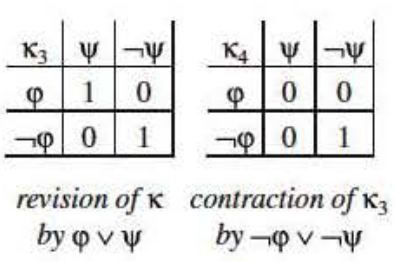

Fig. 2 A Counter-example to Superexpansion?

bomb country $X$, this issue would be settled for Tom, for having learned that country $A$ will bomb country $X$, Tom would be obliged to believe that country $B$ will not bomb country $X$-and this is unreasonable to Tom.

Obviously $(\mathrm{K} * 7)$, or the equivalent statement $(25)$, is violated by this example.

Still, I think we may maintain $(\mathrm{K} * 7)$. Figure 2 below displays a plausible initial epistemic state $\kappa$. Scenarios 1 and 2 are represented by $\kappa_{1}$ and $\kappa_{2}$, which are, more precisely, the $\varphi \rightarrow 1$ - and the $\psi \rightarrow 1$-conditionalization of $\kappa$. However, more complicated things are going on in scenario 3 . Pedersen presents the intelligence officer's information that "country $A$ or country $B$ or both will bomb country $X$ " in a way that suggests that its point is to make clear that the "or" is to be understood inclusively, not exclusively. If the information had been that "either country $A$ or country $B$ (and not both) will bomb country $X$ ", there would be no counter-example, and the supplementary argument in the last paragraph of the quote would not apply; after learning that country $A$ will bomb country $X$, Tom would indeed be confirmed in believing that country $B$ will not bomb country $X$.

However, the communicative function of "or" is more complicated. In general, if I say " $p$ or $q$ ", I express, according to Grice's maxim of quantity, that I believe that $p$ or $q$, but do not believe $p$ and do not believe $q$, and hence exclude neither $p$ nor $q$; otherwise my assertion would have been misleading. And according to Grice's maxim of quality, my evidence is such as to justify the disjunctive belief, but not any stronger one to the effect that $p$, non- $p, q$, or non- $q$.

So, if the officer says " $\varphi$ or $\psi$ or both", the only belief he expresses is indeed the belief in $\varphi \vee \psi$, but he also expresses many non-beliefs, in particular that he excludes neither $\varphi$, nor $\psi$, nor $\varphi \wedge \psi$. And if Tom trusts his officer, he adopts the officer's doxastic attitude, he revises by $\varphi \vee \psi$, and he contracts by $\neg \varphi \vee \neg \psi$, in order not to exclude $\varphi \wedge \psi$. Given the symmetry between $\varphi$ and $\psi$, the other attitudes concerning $\varphi$ and $\psi$ then follow. That is, if Grice's conversational maxims are correctly applied, there is not only a revision going in scenario 3, but also a contraction. And then, of course, there is no counter-example to Superexpansion. This is again displayed in Fig. 2, where $\kappa_{3}$ is the $\varphi \vee \psi \rightarrow 1$-conditionalization of the initial $\kappa$ (in which $\neg \varphi \vee \neg \psi$ is still believed) and $\kappa_{4}$ is the $\neg \varphi \vee \neg \psi \rightarrow 0$-conditionalization of $\kappa_{3}$ (in which $\neg \varphi \vee \neg \psi$ is no longer believed).

Note that these tables assume a symmetry concerning $\varphi$ and $\psi$, concerning the credibility of the attacks of country $A$ and country $B$. We might build in an asymmetry instead, and then the situation would change. 
To confirm my argument above, suppose that in scenario 1 the officer informs Tom that country $A$ will bomb country $X$ or both countries will. The belief thereby expressed is the same as that in the original scenario 1. But why, then, should the officer choose such a convoluted expression? Because he thereby expresses different non-beliefs, namely that he does not exclude that both countries will bomb country $X$. And then, Tom should again contract by $\neg \varphi \vee \neg \psi$. In the original scenario 1 , by contrast, the officer does not say anything about country $B$, and hence Tom may stick to his beliefs about country $B$, as Pedersen has assumed.

We might change scenario 3 in a converse way and suppose that the officer only says that country $A$ or country $B$ will bomb country $X$, without enforcing the inclusive reading of "or" by adding "or both". Then the case seems ambiguous to me. Either Tom might read "or" exclusively and hence stick to his belief that not both countries, $A$ and $B$, will bomb country $X$. Or Tom might guess that the inclusive reading is intended; but then my redescription of the case holds good. Either way, no counterexample to Superexpansion seems to be forthcoming.

\section{The Intersection Postulate for AGM-Contraction}

Let me turn to some of the AGM contraction postulates, which have, it seems, met even more doubt. And let me start with the postulate $(\mathrm{K} * 7)$, Intersection, which says:

(26) $(K \div \varphi) \cap(K \div \psi) \subseteq K \div(\varphi \wedge \psi)$

This corresponds to the revision postulate $(\mathrm{K} * 7)$ just discussed. Sven Ove Hansson has been very active in producing (counter-)examples. In $(1999$, p. 79$)$ he tells a story also consisting of three scenarios and allegedly undermining the plausibility of Intersection:

I believe that Accra is a national capital $(\varphi)$. I also believe that Bangui is a national capital $(\psi)$ As a (logical) consequence of this, I also believe that either Accra or Bangui is a national capital $(\varphi \vee \psi)$.

Case 1: 'Give the name of an African capital' says my geography teacher.

'Accra' I say, confidently.

The teacher looks angrily at me without saying a word. I lose my belief in $\varphi$. However, I still retain my belief in $\psi$, and consequently in $\varphi \vee \psi$.

Case 2: I answer 'Bangui' to the same question. The teacher gives me the same wordless response. In this case, I lose my belief in $\psi$, but I retain my belief in $\varphi$ and consequently my belief in $\varphi \vee \psi$.

Case 3: 'Give the names of two African capitals' says my geography teacher.

‘Accra and Bangui' I say, confidently.

The teacher looks angrily at me without saying a word. I lose confidence in my answer, that is, I lose my belief in $\varphi \wedge \psi$. Since my beliefs in $\varphi$ and in $\psi$ were equally strong, I cannot choose between them, so I lose both of them.

After this, I no longer believe in $\varphi \vee \psi$. 


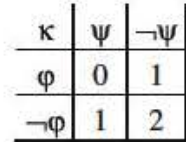

Initial

State

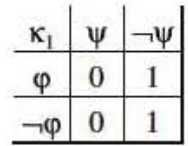

contraction of $\kappa$ by $\varphi$

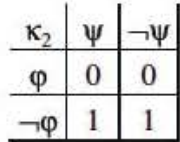

contraction of $\kappa$ by $\psi$

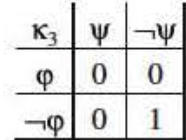

contraction of $\kappa$ by $\varphi \wedge \psi$ or by $[\varphi, \psi]$

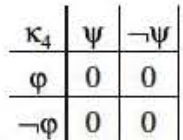

contraction

of $\kappa$ first by $\varphi$ and then by $\psi$

Fig. 3 A Counter-example to Contraction?

At first blush, Hansson's response to case 3 sounds plausible. I suspect, however, this is so because the teacher's angry look is interpreted as, respectively, $\varphi$ and $\psi$ being false. So, if case 1 is actually a revision by $\neg \varphi$, case 2 a revision by $\neg \psi$, and case 3 a revision by $\neg \varphi \wedge \neg \psi$, Hansson's intuitions concerning the retention of $\varphi \vee \psi$ come out right. It is not easy to avoid this interpretation. The intuitive confusion of inner and outer negation - in this case of disbelief and non-belief-is ubiquitous. And the variable interpretation of (dis)belief would make the confusion even worse.

Still, let us assume that the teacher's angry look just makes me insecure so that we are indeed dealing only with contractions. Fig. 3 then describes all possible contractions involved. $\kappa_{1}$ and $\kappa_{2}$ represent the contractions in case 1 and case 2 . These cases are unproblematic.

However, I think that case 3 is again ambiguous. The look might make me uncertain about the whole of my answer. So I contract by $\varphi \wedge \psi$, thus give up $\varphi$ as well as $\psi$ (because I am indifferent between them) and retain $\varphi \vee \psi$. This is represented by $\kappa_{3}$ in Fig. 3 .

It is more plausible, though, that the look makes me uncertain about both parts of my answer. So I contract by $\varphi$ and by $\psi$. This may be understood as what Fuhrmann and Hansson (1994) call package contraction by $[\varphi, \psi]$, in which case I still retain $\varphi \vee \psi$ (according to Fuhrmann and Hansson (1994), and according to my rankingtheoretic reconstruction of multiple and in particular package contraction in Spohn (2010) - for details see there). The result is also represented by $\kappa_{3}$ in Fig. 3. The sameness is accidental; in general, single contraction by $\varphi \wedge \psi$ and package contraction $[\varphi, \psi]$ fall apart.

Or it may be understood as an iterated contraction; I first contract by $\varphi$ and then by $\psi$ (or the other way around). Then the case falls into the uncertainties of AGM belief revision theory vis-à-vis iterated contraction (and revision). Rankingtheoretic contraction, by contrast, can be iterated (for the complete logic of iterated contraction see Hild and Spohn (2008)). And it says that by first contracting by $\varphi$ and then by $\psi$ one ends up with no longer believing $\varphi \vee \psi$ (at least if $\varphi$ and $\psi$ are doxastically independent in the ranking-theoretic sense, as may be plausibly assumed in Hanssons's example). This is represented by $\kappa_{4}$ in Fig. 3. 
Again, these results depend on the built-in symmetries between $\varphi$ and $\psi$ and their independence and thus on the prior state $\kappa$ and its acquisition. If it were different, the contractions might have different results.

Thus, I have offered two different explanations of Hansson's intuition without the need to reject Intersection. In this case, I did not allude to maxims of conversation as in the previous section (since the teacher does not say anything). The effect, however, is similar. Plausibly, other or more complicated belief changes are going on in this example than merely single contractions. Therefore it does not provide any reason to change the postulates characterizing those single contractions.

\section{The Recovery Postulate}

Finally, I turn to the most contested of all contraction postulates, Recovery $(\mathrm{K} \div 5)$, which asserts:

$$
\text { (27) } K \subseteq \operatorname{Cn}((K \div \varphi) \cup\{\varphi\})
$$

Hansson (1999, p. 73) presents the following example: Suppose I am convinced that George is a murderer $(=\psi)$ and hence that George is a criminal $(=\varphi)$; thus $\varphi$, $\psi \in K$. Now I hear the district attorney stating: "We have no evidence whatsoever that George is a criminal." I need not conclude that George is innocent, but certainly I contract by $\varphi$ and thus also lose the belief that $\psi$. Next, I learn that George has been arrested by the police (perhaps because of some minor crime). So, I accept that George is a criminal, after all, i.e., I expand by $\varphi$. Recovery then requires that $\psi \in C n((K \div \varphi) \cup\{\varphi\})$, i.e., that I also return to my belief that George is a murderer. I can do so only because I must have retained the belief in $\varphi \rightarrow \psi$ while giving up the belief in $\varphi$ and thus in $\psi$. But this seems absurd, and hence we face a clear counter-example against Recovery.

This argument is indeed impressive - but not unassailable. First, let me repeat that the ranking-theoretic conditionalization rules are extremely flexible; any standard doxastic movement you might want to describe can be described with them. The only issue is whether the description is natural. However, that is the second point: what is natural is quite unclear. Is the example really intended as a core example of contraction theory, such that one must find a characterization of contraction that directly fits the example? Or may we give more indirect accounts? Do we need, and would we approve of, various axiomatizations of contraction operations, each fitting at least one plausible example? There are no clear rules for this kind of discussion, and as long as this is so the relation between theory and application does not allow any definite conclusions.

Let us look more closely at the example. Makinson (1997) observes (with reference to the so-called filtering condition of Fuhrmann (1991), p. 184) that I believe $\varphi$ (that George is a criminal) only because I believe $\psi$ (that George is a murderer). Hence I believe $\varphi \rightarrow \psi$, too, only because I believe $\psi$, so that by giving up $\varphi$ and hence $\psi$ the belief in $\varphi \rightarrow \psi$ should disappear as well. This implicit appeal 


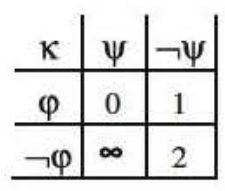

Initial

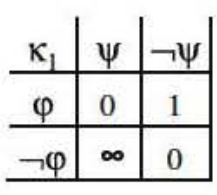

contraction

of $\kappa$ by $\varphi$

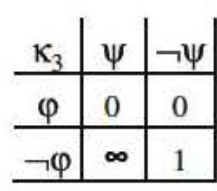

contraction

of $\kappa$ by $\varphi \wedge \psi$

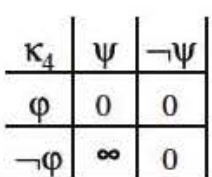

contraction

of $\kappa$ first by $\psi$

and the $n$ by $\varphi$

Fig. 4 A Counter-example to Recovery?

to justificatory relations captures our intuition well and might explain the violation of Recovery (though the "only because" receives no further explication). However, I find the conclusion of Makinson (1997, p. 478) not fully intelligible:

Examples such as those above ... show that even when a theory is taken as closed under consequence, recovery is still an inappropriate condition for the operation of contraction when the theory is seen as comprising not only statements but also a relation or other structural element indicating lines of justification, grounding, or reasons for belief. As soon as contraction makes use of the notion " $y$ is believed only because of $x$ ", we run into counterexamples to recovery ... But when a theory is taken as "naked", i.e. as a bare set of statements closed under consequence, then recovery appears to be free of intuitive counterexamples.

I would have thought that the conclusion is that it does not make much sense to consider "naked" theories, i.e., belief states represented simply as sets of sentences, in relation to contraction, since the example makes clear that contraction is governed by further parameters not contained in that simple representation. This is exactly the conclusion elaborated by Haas (2005, Sect. 2.10).

Inow face a dialectical problem, though. A ranking function is clearly not a naked theory in Makinson's sense. It embodies justificatory relations; whether it does so in a generally acceptable way, and whether it can specifically explicate the "only because", does not really matter. (I am suspicious of the "only because"; we rarely, if ever, believe things only for one reason.) Nevertheless, it is my task to defend Recovery. Indeed, my explanation for our intuitions concerning George is a different one.

First, circumstances might be such that recovery is absolutely right. There might be only one crime under dispute, a murder, and the issue might be whether George has committed it, and not whether George is a more or less dangerous criminal. Thus, I might firmly believe that he is either innocent or a murderer so that, when hearing that the police arrested him, my conclusion is that he is a murderer, after all.

These are special circumstances, though. The generic knowledge about criminals to which the example appeals is different. In my view, we are not dealing here with two sentences or propositions, $\varphi$ and $\psi$, of which one, $\psi$, happens to entail the other, $\varphi$. We are rather dealing with a single scale or variable which, in this simple case, takes only three values: "murderer", "criminal, but not a murderer", and "not criminal". (See Fig. 4, where $\varphi$ and $\psi$ generate a $2 \times 2$ matrix. However, one field 
is impossible and receives negative rank $\infty$; one can't be an innocent murderer. So, you should rather read the remaining three fields as a single, three-valued scale.)

The default for such scales or variables is that a distribution of degrees of belief over the scale is single-peaked. In the case of negative ranks this means that the distribution of negative ranks over the scale has only one local minimum; so, the distribution should rather be called 'single-dented'.

In the present example, the default means: For each person, there is one degree of criminality which is most credible (where credibility is measured here by two-sided ranks, but the default as well applies to other kinds of credibility like probabilities), and other degrees of criminality are the less credible, the further away they are from the most credible degree, i.e., they decrease in a weakly monotonous way. This default is obeyed in my initial doxastic state $\kappa$ displayed in Fig. 4, in which I believe George to be a murderer; there negative ranks take their minimum at the value "murderer" and then increase.

Now, a standard AGM contraction by $\varphi$ (or a $\varphi \rightarrow 0$-conditionalization), as displayed in the second matrix of Fig. 4, produces a two-peaked or 'two-dented' distribution: both "not criminal" and "murderer" receive negative rank 0 and only the middle value ("criminal, but not a murderer" receives a higher negative rank (and remains thus disbelieved). This just reflects the retention of $\varphi \rightarrow \psi$. Thus, AGM contraction violates the default of single-peakedness (or 'single-dentedness').

Precisely for this reason we do not understand the district attorney's message as an invitation for a standard contraction. Rather, I think the message "there is no evidence that George is a criminal" is tacitly supplemented by "let alone a murderer", in conformity to Grice's maxim of quantity. That is, we understand it as an invitation to contract not by $\varphi \wedge \psi$ (as displayed in the third matrix of Fig. 4), but by $\psi$ (George is a murderer), and then, if still necessary, by $\varphi$ or, what comes to the same, by $\varphi \wedge \neg \psi$ (as displayed in the fourth matrix of Fig. 4). In other words, we understand it as an invitation to perform a mild contraction by $\varphi$ in the sense of Levi (2004, p. 142f.), after which no beliefs about George are retained. Given this reinterpretation there is no conflict between Recovery and the example.

Levi (2004, p. 65f.) finds another type of example to be absolutely telling against Recovery (see also his discussion of still another example in Levi (1991), p. 134ff.). Suppose you believe that a certain random experiment has been performed $(=\varphi)$, say, a coin has been thrown, and furthermore you believe in a certain outcome of that experiment $(=\psi)$, say, heads. Now, doubts are raised as to whether the experiment was at all performed. So, you contract by $\varphi$ and thereby give up $\psi$ as well. Suppose, finally, that your doubts are dispelled. So, you again believe in $\varphi$. Levi takes it to be obvious that, in this case, it should be entirely open to you whether or not the random $\psi$ obtains-another violation of Recovery.

I do not find this story so determinate. Again, circumstances might be such that Recovery is appropriate. For instance, the doubt might concern the correct execution of the random experiment; it might have been a fake. Still, there is no doubt about its result, if the experiment is counted as valid. In that case Recovery seems mandatory.

However, I agree with Levi that this is not the normal interpretation of the situation. But I have a different explanation of the normal interpretation. In my view, the point 
of the example is not randomness, but presupposition. $\psi$ presupposes $\varphi$ (in the formal linguistic sense); one cannot speak of the result of an experiment unless the experiment has been performed. And then it seems to be a pragmatic rule that, if the requirement is to withdraw a presupposition, then one has to withdraw the item depending on this presupposition explicitly, and not merely as an effect of giving up the presupposition.

Let us look at the situation a bit more closely. Of course, the issue depends on which formal account of presuppositions to accept. We may say that $q$ (semantically) presupposes $p$ if both $q$ and $\neg q$ logically entail $p$, although $p$ is not logically true; since Strawson (1950) this is standard as a first attempt at semantic presupposition. Then, however, it is clear that our propositional framework, or the sentential framework with its consequence relation $\mathrm{Cn}$, is not suited for formally dealing with presuppositions. Or we may treat presuppositions within dynamic semantics. But again, our framework is not attuned to such alternatives. Hence we have to be content with an informal discussion; it will be good enough.

To begin with, it seems that any argument and hence any belief change concerning $q$ leaves the presupposition $p$ untouched. For instance, if we argue about, and take various attitudes towards, whether or not Jim quit smoking, or whether or not John won the race, all this takes place on the background of the presupposition that he did smoke in the past, or, respectively, that there was a race.

What happens, though, if we argue about the presupposition $p$ itself? I think we may distinguish two cases then, instantiated by the two examples just given. Let us look at the first example and suppose that I believe that Jim quit smoking and hence smoked in the past. Now doubts are raised that Jim smoked in the past, and maybe I accept these doubts. What happens then to my belief that Jim quit smoking? Well, why did I have this belief in the first place? Presumably, because I haven't seen Jim smoking for quite a while and because I thought to remember to have often seen him smoking in the past. It is characteristic of this example that "Jim quit smoking" can be decomposed into two logically independent sentences "Jim smoked in the past" and "Jim does not smoke now". Hence, if I am to give up that Jim smoked in the past, I have to give up "Jim quit smoking" as well, but I will retain "Jim does not smoke now". This entails, however, that, if the doubts are dispelled and I return to my belief that Jim smoked in the past, I will also return to my belief that Jim quit smoking, since I retained the belief that Jim does not smoke now. And so we have a case of Recovery.

However, this characteristic does not always hold. Let us look at a second example, where $q=$ "John won the race", which presupposes $p=$ "there was a race". Again, assume that I believe both and that doubts are raised about the presupposition. The point now is "John won the race" is not decomposable in the way above. It is usually very unclear what John is supposed to have done if there was no race at all, what it is apart from the presupposition that is correctly described as John's winning the race (with the help of the presupposition). So, in this case doubts about the presupposition are at the same time doubts about John's having done anything that could be described as winning the race in the case there should have been a race. If so, the withdrawal of the presupposition $p$ must be accompanied by an explicit withdrawal of $q$, so that 
the material implication $p \rightarrow q$ is lost as well. Again, we have no counter-example against Recovery; Recovery does not apply at all, because a more complex doxastic change has taken place in the second example. And it seems to me that, at least under the normal interpretation, Levi's example of the random experiment is of the second characteristic. If the coin has not been thrown at all, there is no behavior of the coin that could be described as the coin's showing head in case it had been thrown.

So, the pragmatic rule stated above seems to apply at least to the second kind of example characterized by the non-decomposability of presupposition and content. This pragmatic rule is quite different from my above observation about scales. The pragmatic effect, however, is the same. And again this effect agrees with Levi's mild contraction. Note, by the way, that what I described as special circumstances in the criminal and the random example above can easily be reconciled with mild contraction; informational loss is plausibly distributed under these circumstances in such a way that mild contraction and AGM contraction arrive at the same result.

Hence I entirely agree with Levi on the description of the examples. I disagree on their explanation. Levi feels urged to postulate another kind of contraction operation governed by different axioms, and Makinson has the hunch that taking account of justificatory relations will lead to such a different contraction operation. By contrast, I find AGM contraction sufficient on the theoretical level and invoke various pragmatic principles explaining why more complex things might be going on in certain situations than single AGM contractions.

\section{Conclusion}

All in all, I feel justified in repeating the conclusions already sketched in the introduction. First, ranking-theoretic conditionalization includes expansion, revisions, and contraction as special cases. And since the latter can plausibly be explicated by ranking theory only in the way specified in Sect.3, this entails that the standard AGM postulates $(\mathrm{K} * 1)-(\mathrm{K} * 8)$ and $(\mathrm{K} \div 1)-(\mathrm{K} \div 8)$ must hold for revisions and contractions. However, because of its much larger generality (which in turn is due to the additional structure assumed in ranking theory) ranking-theoretic conditionalization has resources to cope with other kinds of examples and with more kinds of belief change than the standard AGM theory. On a theoretical level ranking-theoretic conditionalization is all we need.

The second conclusion is more important. I did not, and did not attempt to, offer any systematic account for dealing with all kinds of examples. On the contrary, I intentionally used a variegated bunch of pragmatic and interpretational strategies for coping with the examples. I believe that all these strategies, and certainly more, are actually applied. So there is no reasonable hope for a unified treatment of the examples. Rather, we must study all the pragmatic and interpretational ways in systematic detail. (Cf., e.g., Merin 1999, 2003a, b, who has made various interesting and relevant observations concerning the formal pragmatics of presuppositions and scale phenomena, though not in direct connection to belief revision.) And we must study 
the interaction of those strategies. I see here a potentially very rich, but so far little explored research field at the interface between linguistics and formal epistemology. In a way, the gist of the paper was at least to point at this large research field.

And the third conclusion is immediate: If this large research field interferes, there can be no direct argument from intuitions about examples to the basic axioms of belief change; there is always large space for alternative explanations of the intuitions within this interfering field. Hence, I have little sympathy for experimenting with these basic axioms. Rather, these axioms have theoretical justifications, which are amply provided within ranking theory (see Spohn 2012, Chaps. 5 and 8). These theoretical justifications are the important ones, and hence I stand by the standard AGM axioms unshaken.

\section{References}

Alchourrón, C. E., Gärdenfors, P., \& Makinson, D. (1985). On the logic of theory change: Partial meet functions for contraction and revision. Journal of Symbolic Logic, 50, 510-530.

Cohen, L. J. (1970). The implications of induction. London: Methuen.

Cohen, L. J. (1980). Some historical remarks on the Baconian conception of probability. Journal of the History of Ideas, 41, 219-231.

Dubois, D., \& Prade, H. (1988). Possibility theory: An approach to computerized processing of uncertainty. New York: Plenum Press.

Field, H. (1978). A note on Jeffrey conditionalization. Philosophy of Science, 45, 361-367.

Fuhrmann, André. (1991). Theory contraction through base contraction. Journal of Philosophical Logic, 20, 175-203.

Fuhrmann, A., \& Hansson, S. O. (1994). A survey of multiple contractions. Journal of Logic, Language, and Information, 3, 39-76.

Gärdenfors, P. (1988). Knowledge in flux. Cambridge, MA: MIT Press.

Haas, G. (2005). Revision und Rechtfertigung. Eine Theorie der Theorieänderung, Heidelberg: Synchron Wissenschaftsverlag der Autoren.

Hansson, S. O. (1997). Special issue on non-prioritized belief revision. Theoria, 63, 1-134.

Hansson, S. O. (1999). Theory change and database updating. A Textbook of Belief Dynamics. Dordrecht: Kluwer.

Hild, M., \& Spohn, W. (2008). The measurement of ranks and the laws of iterated contraction. Artificial Intelligence, 172, 1195-1218.

Huber, F. (2006). Ranking functions and rankings on languages. Artificial Intelligence, 170, 462471.

Jeffrey, R. C. (1965/1983). The logic of decision (2 ${ }^{\text {nd }}$ ed.). Chicago: University of Chicago Press. Levi, I. (1991). The fixation of belief and its undoing. Cambridge: Cambridge University Press.

Levi, I. (2004). Mild contraction: Evaluating loss of information due to loss of belief. Oxford: Oxford University Press.

Makinson, D. (1997a). Screened revision. Theoria, 63, 14-23.

Makinson, D., et al. (1997b). On the force of some apparent counterexamples to recovery. In E. G. Valdés (Ed.), Normative systems in legal and moral theory (pp. 475-481). Festschrift for Carlos Alchourrón and Eugenio Bulygin, Berlin: Duncker \& Humblot.

Merin, A. (1999). Information, relevance and social decision-making: Some principles and results of decision-theoretic semantics. In L. S. Moss, J. Ginzburg, \& M. De Rijke (Eds.), Logic, Language, and Computation (Vol. 2, pp. 179-221). Stanford, CA: CSLI Publications (Online at http://www. semanticsarchive.net) 
Merin, A. (2003a). Replacing horn scales by act-based relevance orderings to keep negation and numerals meaningful. Forschungsberichte der DFG-Forschergruppe Logik in der Philosophie, No. 110, University of Konstanz. (Online at http://www.semanticsarchive.net)

Merin, A. (2003b). Presuppositions and practical reason: A study in decision-theoretic semantics. Forschungsberichte der DFG-Forschergruppe Logik in der Philosophie, No. 114, University of Konstanz. (Online at http://www.semanticsarchive.net)

Rabinowicz, W. (1996). Stable revision, or is preservation worth preserving?. In A. Fuhrmann \& H. Rott (Eds.), Logic, action, and information (pp. 101-128). de Gruyter: Essays on Logic in Philosophy and Artificial Intelligence, Berlin.

Rescher, N. (1964). Hypothetical reasoning. Amsterdam: North-Holland.

Rott, H. (1989). Conditionals and theory change: Revisions expansions and additions. Synthese, 81, 91-113.

Rott, H. (1999). Coherence and conservatism in the dynamics of belief Part I: Finding the right framework. Erkenntnis, 50, 387-412.

Shackle, G. L. S. (1961). Decision, order, and time in human affairs ( $2^{\text {nd }}$ ed.). Cambridge: Cambridge University Press.

Shenoy, P. P. (1991). On Spohn's rule for revision of beliefs. International Journal of Approximate Reasoning, 5, 149-181.

Spohn, W. (1983). Eine Theorie der Kausalität, unpublished Habilitationsschrift, Universität München, pdf-version at: http://www.uni-konstanz.de/FuF/Philo/Philosophie/philosophie/files/ habilitation.pdf

Spohn, W. (1988). Ordinal conditional functions. A dynamic theory of epistemic states. In W. L. Harper \& B. Skyrms (Eds.), Causation in decision, belief change, and statistics (Vol. II, pp. 105-134). Dordrecht: Kluwer.

Spohn, W. (2010). Multiple contraction revisited. In M. Suárez, M. Dorato, \& M. Rédei (Eds.), EPSA epistemology and methodology of science (Vol. 1, pp. 279-288).Launch of the European Philosophy of Science Association, Dordrecht: Springer.

Spohn, W. (2012). The laws of belief. Ranking Theory and Its Philosophical Applications, Oxford: Oxford University Press.

Strawson, P. F. (1950). On referring. Mind, 59, 320-344.

Teller, P. (1976). Conditionalization, observation and change of preference. In W. L. Harper \& C. A. Hooker (Eds.), Foundations of Probability Theory (pp. 205-259). Reidel, Dordrecht: Statistical Inference and Statistical Theories of Science.

Zadeh, Lofti A. (1978). Fuzzy sets as a basis for a theory of possibility. Fuzzy Sets and Systems, 1, 3-28. 